\title{
SARS-CoV-2 variants genome analysis of Indonesian isolates and their responses to available vaccines
}

\section{Dyana Yurico}

Swiss German University

Michael Jeremy

Swiss German University

Nasser Mohamed Ghassan Mohamed Adnan Shaikho

Swiss German University

Kholis Abdurachim Audah ( $\sim$ kholis.audah@sgu.ac.id )

Swiss German University

\section{Research Article}

Keywords:

Posted Date: January 11th, 2022

DOI: https://doi.org/10.21203/rs.3.rs-1225245/v1

License: @ (i) This work is licensed under a Creative Commons Attribution 4.0 International License. Read Full License 


\section{Abstract \\ Background}

SARS-CoV-2 is a virus that initially appeared in Wuhan, China, at the end of 2019. Since then, the virus has spread until to almost all countries resulting in a global pandemic. Over time, this virus continues to mutate and produce several other variants. In Indonesia, there are multiple variants of SARS-CoV-2 identified, as well as various local variants that are not yet considered to be 'variants of concern'. Therefore, this investigation is intended to understand the prevalence and epidemiology of the virus, along with detecting the mutations that occur in genes associated with whole-genome-sequences (WGS) isolated in Indonesia.

\section{Result}

Analyses were performed to investigate SARS-CoV-2 prevalence in Indonesia using data obtained from GISAID.org. A wholegenome sequencing was performed on the random samples taken from GISAID.org utilizing the BLAST tool from NCBI. The variants identified in Indonesia are Alpha, Beta and Delta variants, as well as local variants B.1.470 and B.1.466.2. As of the end of November, it was found that there are a total of 5.348 cases of the Delta, 78 cases of the Alpha, 22 cases of the Beta, 572 cases of the local variant B.1.470, and 1.833 cases of the local variant B.1.466.2. Other cases include 219 cases of local variant B.1.1.398, 160 cases of local variant B.1.459 and 1.028 cases of the wild type. In total there are 9.260 isolated genomes collected in GISAID that are located in Indonesia. Using BLAST, WGS of Alpha, Delta, Beta, B.1.470 and B.1.466.2 variants isolated in Indonesia was compared with the wild type from Wuhan NC.045512.2. It was found that multiple mutations have occurred in the samples. The mutations identified as are H69del, V70I, N501Y, D614G, A570D, P681H, T716I, S982A, and D1118H in the Alpha variant, T19R, L452R, T478K, D614G, and D950N in the Delta variant, D215G, D614G, A701V, L241-, L242-, K417N in the Beta variant, D614G, L242F, and S12F in the B.1.470 variant and D614G, N439K, and P681R in the B.1.466.2 variant. These mutations had caused alterations in the characteristics of the virus and how it may affect vaccine efficacy.

\section{Conclusions}

The results from whole-genome sequencing of variants isolated in Indonesia have found that multiple mutations have occurred in genes of the SARS-CoV-2 Virus and it caused alterations in the characteristics of the virus and may affect vaccine efficacy. It should be noted that classification from the GISAID website may change overtime. The result in this paper is based on the data taken at the end of November.

\section{Background}

Towards the end of the year 2019, China reported its first case of a type of infectious disease in Wuhan. Since then, this disease, a type of coronavirus called COVID-19, has caused an ongoing global pandemic. Three bronchoalveolar lavage samples were collected from a patient with pneumonia with unknown etiology, and these samples were analyzed using polymerase chain reaction or PCR, showing a positive infection for pan-Betacoronavirus (WHO, 2020). The very first whole genome sequence (WGS) of the virus was then obtained using Illumina and nanopore sequencing. This sequence is known as the Wild Type (WT) WGS of SARS-CoV-2. Bioinformatics analysis using the phylogenetic tree showed that the virus had similar features with the coronavirus family and that the virus belonged to the Betacoronavirus 2B lineage (WHO, 2020). Using sequence alignment, it was identified that the COVID-19 virus had resemblance with SARS for example, had many similarities with another coronavirus strain, BatCov RaTG13 (Clinic, 2021). 
Symptoms from COVID-19 are fever, dry cough, and tiredness. Other symptoms include aches and pains, sore throat, diarrhea, headache, loss of taste or smell. Serious symptoms include shortness of breath or difficulty in breathing, chest pain or pressure, loss of speech and inability to move. These serious symptoms are the indicators that the person should seek medical attention immediately. The fatality of this virus depends on various factors, like age, whether a person has hereditary diseases or not. The fatality ratio increases as age increases.

In early March 2020, Sars-CoV2 began to enter Indonesia, initially detected in Depok, West Java (Wiranti Wiranti, Ayun Sriatmi, 2020). As of November 2021, there are already seven Sars-CoV2 variants worldwide, including the Alpha variant, Beta variant, Delta variant, Gamma variant, Lambda variant, MU variant and OMICRON variant (Tracking SARS-CoV-2 Variants, n.d). However, only three variants that have been seen in Indonesia, those being the Alpha, Beta, and Delta variants. In addition, various local variants were also identified in Indonesia, such as B.1.470, B.1.466.2, B.1.1.398 and B.1.459, with both B.1.470 and B.1.466.2 enlisted as Indonesian lineages by PANGO (Fibriani et al., 2021). Both enlisted Indonesian lineages are not yet considered as 'variants of concern', however the variant B.1.466.2 is currently being observed by the World Health Organization enlisted as 'Alerts for Further Monitoring (World Health Organization, n.d.; Fibriani et al., 2021).

\section{Methods And Materials}

The materials used in this study were obtained from GISAID.org. The search was filtered by selecting Indonesia as the location to obtain whole genome sequences of Indonesia isolates. The Wild Type WGS from Wuhan was obtained from NCBI.

The WGS from Indonesia was then compared with the WGS Wild Type from Wuhan. Afterwards, the similarity between was obtained, along with the mutations in the nucleotide sequences and the amino acids in the variants. The comparison was done using the BLAST tool from NCBI. To obtain the prevalence in Indonesia, the data was obtained from GISAID.org.

\section{Results And Discussion}

In order to be able to deal with SARS-CoV-2, a better understanding of its structure and domains is important. The first part of an infection is the viral entry, wherein the virus comes into contact with the host cell. For viral entry to occur, SARS-CoV-2 targets the angiotensin-converting enzyme 2 (ACE2) receptors through the use of the receptor-binding domain (RBD) that is

located in the spike protein of the virus (Mittal et al., 2020). By doing this, the virus is able to fuse to the host-cell membrane and start spreading.

The COVID-19 virus along with its variants are single-stranded RNA that are non-segmented and have the range of 26 to 32 $\mathrm{kb}$ (Mittal et al., 2020). Moreover, the virus is a spherical shape that is covered with spikes responsible for the transmission of the virus. The diameter of virus ranges anywhere from $60 \mathrm{~nm}$ to $140 \mathrm{~nm}$, with the spikes ranging from $9 \mathrm{~nm}$ to $12 \mathrm{~nm}$ respectively (Mittal et al., 2020).

As for the position of the structures for the sequence of SARS-CoV-2, the official reference sequence hCoV19/Wuhan/WIV04/2019 (WIV04) isolated in Wuhan China will be utilized. For an elevated understanding, the nucleotide positions of each structure will be provided. The genome starts with ORF1a and ORF1b which overlap one another, with ORF1a having the position 266 to 13468, and ORF1b having the position 266 to 21555 in the sequence (Gisaid, n.d.). The next structure is the $S$ structure that corresponds to the spike protein of SARS-CoV-2, and it has the position of 21563 to 25384. Next in the sequence are ORF3a and ORF3b, which can be found in position 25393 to 26220 for ORF3a and 25765 to 26220 for ORF3b respectively; ORF3a corresponds to nonstructural protein NS3a, whereas ORF3b corresponds to NS3b.

Next, the E structure corresponding to the envelope protein can be seen in the position 26245 to 26472 . Another structure follows, that being the structure M corresponding to the membrane protein and is positioned at 26523 to 27191 . After that, 
ORF6 can be seen located at position 27202 to 27387 , which corresponds to nonstructural protein NS6. Then, ORF7a and ORF7b follow, with ORF7a at position 27394 to 27759 and corresponding to nonstructural protein NS7a, and ORF7b at position 27756 to 27887 and corresponding to nonstructural protein NS7b. ORF8 then follows, positioned at 27894 to 28259 and corresponding to nonstructural protein NS8. Next, the structure N, which corresponds to the nucleocapsid protein, can be seen at position 28274 to 29533.

Within the range of the $\mathrm{N}$ structure, ORF9a and ORF9b can be found in the positions 28284 to 28577 and 28734 to 28955 respectively; ORF9a corresponds to nonstructural protein NS9a, whereas ORF9b corresponds to nonstructural protein NS9b. And finally, ORF10 is positioned at 29558 to 29674 , and corresponds to nonstructural protein NS10; currently there is no evidence to suggest that this protein plays a role in the transmission or replication of the virus. The structure of the SARSCoV-2 virus is a 5'-capped and 3'-polyadenylated sequence, and within this sequence, various open reading frames (ORFs) can be found along with 2 untranslated regions (UTRs) (Mittal et al., 2020). These ORFs are responsible for encoding proteins. Moreover, the arrangement of the genome starts with 5'-UTR-replicase genes (ORF1ab) structure proteins S, E, M, and $\mathrm{N}$ can be seen as well as accessory proteins-noncoding 3'-UTR (Mittal et al., 2020). The ORF1a and ORF1ab located at the $5^{\prime}$-end of the genome of SARS-CoV-2 is responsible for 15 nonstructural proteins (Wu et al., 2020). These overlapping ORFs produce 2 polypeptides due to ribosomal frameshifting that occurs, those being pp1a and pp1ab, and the genome encodes for proteases PL2pro along with 3CLpro to cleave said polypeptides to produce 15 nsps (non-segmented proteins), with some of these enzymes produced being highly important for transcription as well as the replication of viral RNA (Mittal et al., 2020). Among the structures of SARS-CoV-2, E is the smallest and is responsible for targeting the endoplasmic reticulum and Golgi complex within the host cells (Nieto-Torres et al., 2011). As for the M protein, it is responsible for viral assembly, being at the center of it (Masters, 2006). The N protein is responsible for viral genome packaging (Mittal et al., 2020). And lastly, the S protein is responsible for viral attachment and entry into a host-cell.

In this study, the differences between the variants was analyzed using blast from NCBI BLAST. Using this, the identical percentage was then measured, as well as the location of the mutations between the different genome sequences. The variants isolated in Indonesia were selected randomly from the GISAID website. Alpha variant with the accession ID of EPI_ISL_3138832, Beta Variant (EPI_ISL_2434175), and Delta variant (EPI_ISL_5462140) of Sars-CoV2 whole genome sequences in Indonesia were obtained from GISAID database, while the original genome sequence of Sars-CoV2 isolated from Wuhan, China was obtained from the NCBI database (NCBI NC_045512.2). The local variants not yet considered 'variants of concern' were also obtained from GISAID database; B.1.470 Indonesian local variant (EPI_ISL_2854771) and B.1.466.2 Indonesian local variant (EPI_ISL_6827341).

Graphs 1 to 3 show total cases of variant isolates that were sequenced in Indonesia with a period of time of every three months, starting from March 1, 2020 until November 30, 2021. It can be concluded from the graph above, from March 1, 2020 until February 28, 2021, no variant isolated were sequenced in Indonesia. The first graph shows the Alpha variant isolates that were sequenced starting from early March 2021, with 23 isolates being sequenced. An increase in Alpha variant isolates was then observed in the months that followed, from June 2021 until August 2021 with a total of 42 isolates sequenced, with the isolate sequencing showing a decrease in the months to follow, from September 2021 until November 30, 2021 with a total of 13 isolates sequenced.

In the second graph, the sequencing for Beta variants can be observed. The graph shows that the sequencing of the Beta variant in Indonesia started from early March, 2021. From March 1, 2021 until May 30, 2021, 4 isolated sequences for the Beta variants were recorded, and this number started increasing in June 2021 until 31 August 2021 with 13 isolated sequences, and later on decreased in September 2021 until November 30, 2021 with 5 isolated sequences.

The third graph shows Delta variants isolated sequences in Indonesia that started being sequenced in Indonesia with 33 isolates during the month of March 2021 until May 2021. This number went up drastically in June 2021 until August 2021 
with a total of 2,230 isolated sequences and continued to increase in September 2021 until November 30, 2021 with 3,085 more isolated variants.

Table 1

Percentage of variants in Indonesia: (PREVALENCE)

\begin{tabular}{|llll|}
\hline Database & Variant & Total & Percentage \\
\hline GISAID & Delta Variant & 5,348 & $57.75 \%$ \\
\cline { 2 - 4 } & Alpha Variant & 78 & $0.84 \%$ \\
\cline { 2 - 4 } & Beta Variant & 22 & $0.24 \%$ \\
\cline { 2 - 4 } & Gamma Variant & 0 & $0 \%$ \\
\hline Lambda Variant & 0 & $0 \%$ \\
\hline MU Variant & 0 & $0 \%$ \\
\cline { 2 - 4 } & Local Variant B.1.470 & 572 & $6.18 \%$ \\
\cline { 2 - 4 } & Local Variant B.1.466.2 & 1,833 & $19.79 \%$ \\
\cline { 2 - 4 } & Local Variant B.1.1.398 & 219 & $2.37 \%$ \\
\cline { 2 - 4 } & Local Variant B.1.459 & 160 & $1.73 \%$ \\
\hline Wild-Type & 1,028 & $11.10 \%$ \\
\hline Total Isolated WGS in Indonesia & 9,260 & \\
\hline
\end{tabular}

\section{Comparison of WGS using NCBI Blast}

Using NCBI BLAST, we were able to align sequences to one another for comparison, with the differences between the genome sequences being obtained. Using the NCBI BLAST, the reference genome obtained from NCBI NC_045512.2 Wuhan genome was compared with the wild type in Indonesia and the variants that were present in Indonesia (as of November 30, 2021) mentioned in the previous section. When the Indonesian Alpha Variant EPI_ISL_3138832 obtained from GISAID was compared with the reference genome, the sequence alignment showed some mutations and changes in the amino acids between the two sequences. The identical percentage between them was seen to be $99.82 \%$. This was performed for the Beta, Delta, and local variants respectively.

\section{Sequence Comparison between Alpha Variant Isolate in Indonesia with WT from Wuhan}

The Alpha variant of SARS-CoV-2 B.1.1.7 was first identified in the UK. The BLAST NCBI was used to compare the Alpha variant EPI_ISL_3138832 with the WT from Wuhan. From this comparison, it was seen that the Alpha variant EPI_ISL_3138832 from Indonesia had accumulated around 20 mutations which can be seen in Table 4 in the Appendix. Out of these mutations, four mutations have the most potential to influence the characteristics of the virus. These four mutations include 168-H69del, V70I, N501Y, and P681H. The mutation N501Y was observed to occur as the nucleotide A changes to T. According to (Starr et al., 2020; Makowski et al., 2021), it was found that the N501Y mutation increases the affinity of spike protein to bind to the hACE2 receptor and thereby increasing the transmission rate of the virus 
The $\mathrm{P} 681 \mathrm{H}$ was also observed. This mutation occurs due to the change of nucleotide $\mathrm{C}$ to $\mathrm{A}$. This mutation occurs at the proteolytic cleavage site S protein where it increases the cleavage of S1/S2 junction (Lubinski et al., 2021). However, it is still unclear whether this affects the infectivity and membrane fusion rate. Another mutation that was of significance and was present is A570D in the surface glycoprotein, where the nucleotide $C$ changes to $A$. This mutation induces significant conformational destabilization of the virus (Socher et al., 2021).

Table 2. Detectable Mutations (La Rosa et al., 2021)

\begin{tabular}{|c|c|c|c|c|c|c|c|}
\hline \multicolumn{8}{|c|}{ Table 2. Mutations detectable } \\
\hline \multicolumn{2}{|c|}{ Alpha (B.1.1.7) } & \multicolumn{2}{|c|}{ Beta (B.1.351) } & \multicolumn{2}{|c|}{ Delta (B. 1.617.2) } & \multicolumn{2}{|c|}{$\begin{array}{c}\text { Indonesian Local } \\
\text { Variant }\end{array}$} \\
\hline Mutation & $\%$ & Mutation & $\%$ & Mutation & $\%$ & Mutation & $\%$ \\
\hline H69del & 94 & $\mathrm{D} 80 \mathrm{~A}$ & 87 & K77T & 0.8 & D614G & \\
\hline V70del & 95 & D215G & 85 & T95I & 41 & P323L & \\
\hline Y144del & 94 & L241- & 77 & G142D & 54 & Q57H & \\
\hline E484K & 0.2 & L242- & 78 & E156G & 88 & T77A & \\
\hline S494P & 0.3 & L243- & 77 & F157- & 89 & T350। & \\
\hline N501Y & 97 & P384L & 1.2 & R158- & 88 & S126L & \\
\hline \multirow[t]{4}{*}{ A570D } & 98 & K417N & 88 & W258L & 0.2 & G142D & \\
\hline & & E484K & 87 & $\mathrm{~K} 417 \mathrm{~N}$ & 0.4 & P681R & \\
\hline & & N501Y & 87 & L452R & 97 & N439K & \\
\hline & & E516Q & 0.3 & T478K & 97 & & \\
\hline
\end{tabular}

Percentage represent the frequency of mutation occurrence in each variant according to the GISAID Data

No previous study was conducted for the frequency of mutation occurrence in the Indonesian Local Variant

Another mutation observed was in the 982nd position where the amino acid $\mathrm{S}$ changed to $\mathrm{A}$ due to a change in nucleotide from $T$ to $G$. The effect of this substitution results in the intermolecular hydrogen bonding potential between $S$ protein subunits being missing in the Alpha variant (Ostrov, 2021). The D1118H mutation is another mutation that was observed, which occurs in the Alpha variant due to the change of nucleotide $\mathrm{G}$ to $\mathrm{C}$. This mutation is present in the S2 subunit in the $\mathrm{S}$ protein (Chrysostomou et al., 2021). It has been suggested that this mutation can alter the stability and dynamics of trimer assembly (Zhao et al., 2021). These mutations majorly occur within the S protein. The mutations that occur in the gene encoding the $S$ protein can change how the virus interacts with the hACE2 receptor in humans, thereby increasing the infection rate. However, to date, the Alpha variant appears to have little impact on the effectiveness of vaccines.

\section{Sequence Comparison between Delta Variant Isolate in Indonesia with WT from Wuhan}

The SARS-CoV-2 B.1.617.2 Delta variant was first identified in India. This Delta variant contains 3 sub-lineages, B.1.617.1, B.1.617.2, and B.1.617.3. From the comparison between the WT from Wuhan, it can be seen that the Delta variant contains several mutations throughout its genome, which can be seen in Table 5 in the Appendix. The identical percentage between the Delta variant and the reference variant was seen to be $99.90 \%$. Of all the mutations, some mutations observed raise concerns, those being D614G, T478K, L452R and P681R. It can be seen that a specific mutation in the nucleotide from $\mathrm{C}$ to $G$ changed the amino acid from Proline $(P)$ to Arginine (R), P681R. This specific mutation found within the furin cleavage site of the Delta variant causes an enhancement of Delta variant replication through an increase of S1/S2 cleavage (Liu et al., 2021).

Another mutation can be seen at L452R in the Delta variant when the nucleotide changed from $\mathrm{T}$ to $\mathrm{G}$. This mutation causes a higher affinity for the $S$ protein towards the ACE2 receptor, and causes a decrease in the recognition capability of the immune system (Mohammadi et al., 2021). Another mutation from A to $G$ causing the amino acid to change from $D$ to 
$\mathrm{G}$ in 614, D614G. The final concerning mutation in the Delta variant is the mutation from $\mathrm{C}$ to $\mathrm{A}$ which causes the change from $\mathrm{T}$ to $\mathrm{K}$ in T478K. All these mutations could explain how the Delta variant becomes increasingly more easily spread and becomes the highest number of variants that infect people in Indonesia.

\section{Sequence Comparison between Beta Variant Isolate in Indonesia with WT from Wuhan}

The Beta variant was initially discovered in South Africa. The comparison of the Beta variant EPI_ISL_2434175 from Indonesia isolate with the WT from Wuhan as the reference genome showed multiple mutations across various domains in the genome.

The mutations observed can be seen in Table 6 in the Appendix. The identical percentage between them was seen to be 99.89\%. Utilizing the Blast NCBI, a comparison of the Beta variant isolated from Indonesia and the WT from Wuhan was conducted to analyze mutations. Out of all the mutations observed, four mutations detected have the most potential to influence the characteristics of the virus, those being D215G, L241-, L242-, and K417N. The first mutation to be discussed is D215G, which occurs as the nucleotide A changes to G. This mutation, located in the N-terminal domain of the spike protein, results in a change in the amino acid produced, from Aspartic acid (D) to Glycine (G) (Tegally et al., 2021). The next mutation observed is L241-. Here, nucleotides TTACTT are deleted in the Beta variant, resulting in the deletion of the amino acid Leucine (L). Just like L241-, L242- has the nucleotides TTACTT deleted, once again resulting in the deletion of amino acid Leucine (L).

Both L241- and L242- were also observed in the spike protein, located in the N-terminal domain (Tegally et al., 2021). And finally, the last mutation of concern observed was $\mathrm{K} 417 \mathrm{~N}$. This mutation once again occurs in the spike protein, and it occurs when the $G$ nucleotide becomes $T$, resulting in a change in the amino acid from Lysine $(K)$ to Asparagine $(N)$. The $\mathrm{K} 417 \mathrm{~N}$ has more potential to influence the virus due to the substitution being at key residues of the receptor binding domain of the spike protein, and this region can be used for the binding of neutralizing antibodies (Tegally et al., 2021). Moreover, $\mathrm{K} 417 \mathrm{~N}$ results in the tightening of the interactions of the receptor binding domain (RBD) with the cellular receptor angiotensin-converting enzyme 2 (ACE2) (Sanches et al., 2021). This mutation at 417 results in an increase in the interaction of the ACE2 with the amino acid substitution Asparagine $(\mathrm{N})$.

\section{Sequence Comparison between Local Variant Isolates in Indonesia with WT from Wuhan}

Table 7 in the supplementary files shows the comparison between the local variant in Indonesia Isolate EPI_ISL_2854771 that is identified B.1.470 with the reference genome WT from Wuhan. The identical percentage between them was seen to be $99.87 \%$. The Indonesian local variant had in total 12 synonymous mutations, and 18 non-synonymous mutations. The spike protein in total had 3 mutations which include: S12F, L242F and D614G. The D614G mutation was also found in this Indonesian isolate caused by the mutation of nucleotide A to G. This mutation, which can also be found in the Delta and Beta variants, increases infectivity and enhances the replication of the SARS-CoV-2 Virus in the upper respiratory tract (Plante et al., 2021), inducing conformational change in the S1 subunit of S protein (Yurkovetskiy et al., 2020), as well as in the SD2 domain where it enhances the cleavage of furin at the S1/S2 domain junction (Gobeil et al., 2021).

Another observed mutation is S12F, present on the hydrophobic residues in the signal peptide sequence. This mutation occurs due to a change in the nucleotide sequence from $\mathrm{C}$ to $\mathrm{T}$, and has been theorized to affect the entry of the spike glycoprotein into the endoplasmic reticulum (Timmers et al., 2021). Another observed mutation in the spike protein is the L242F due to the change in nucleotide from C to T. T2051 was another mutation seen in the nucleocapsid protein due to a change from C to T. The ORF3a protein was seen to have some mutations as well, those being: D155H, Q57H, S220I. This 
genome was also seen to contain the deletion of SGF in the ORF1a/ ORF1ab polyprotein which was also present in the Alpha variant.

The last comparison made in this review paper was the comparison between the local variant in Indonesia Isolate EPI_ISL_2854771 that is identified as B.1.466.2, with the reference genome WT from Wuhan which can be seen in Table 8 in the Appendix. The identical percentage between them was seen to be $99.83 \%$. The D614G mutation was also found in this isolate, similar to the other variants. Another key mutation observed was N439K. This mutation is able to produce a salt bridge with Glu329 of the hACE2 receptor, with the salt bridge prompting a greater electrostatic complementarity. This mutation also creates a weak salt bridge with Asp442 of RBD. Another mutation, N439K, was observed. N439K causes the bounding to the hACE2 receptor with a higher affinity than the wild-type, which may lead to more infection. In addition, this mutation causes resistance to the SARS-CoV-2 neutralizing antibody REGN10987. This may lead to the failure of neutralization. (Zhou, W. et al.. 2021). Another mutation found in this local variant was P681R which was also found in the Delta variant before. All these mutations were located in the spike protein. Other mutations found in this variant were S206T, T250I, Q57H, I2N, P822L, S126L, T350I.

Table 3. Isolates under study and results obtained by BLAST NCBI

\section{Responses to Available Vaccines}

As of writing this paper, there are 6 approved COVID-19 vaccines, those being; Pfizer-BioNTech (BNT162b2), Moderna (mRNA-1273), Oxford/AstraZeneca (AZD1222), J\&J/Janssen's (Ad26.COV2.S), Novavax (NVX-CoV2373), Sinovac, and Sinopharm. These vaccines differ from one another in what type of vaccine they are; Pfizer-BioNTech and Moderna are mRNA vaccines, Oxford.AstraZeneca and J\&J/Janssen's are viral vector vaccines, Novavax is a recombinant spike vaccine, and Sinovac and Sinopharm are inactivated vaccines (Cevik et al., 2021). All of these vaccines were based on the wild-type virus sequenced early on during the pandemic (in January 2020) and as such their efficacy and effectiveness towards the newer variants is of utmost importance.

It must be noted that the efficacy of different vaccines cannot truly be compared to one another since the testing conducted depends on the clinical environment, studied population, geographical location, among others (Cevik et al., 2021). As well as this, the real time effectiveness of vaccines may prove to be challenging due to various factors such as the certain variant transmissibility, population vaccination status, among others. Yet another aspect to consider is the initial antibody responses, which may help with the overall picture of understanding the efficacy and effectiveness of the various vaccines to the various variants of SARS-CoV-2 (Cevik et al., 2021). And finally, it may prove difficult to assess the efficacy and effectiveness as T cells play a major role in protecting the body from infections (Cevik et al., 2021).

In order to understand the efficacy and effectiveness of the vaccines towards the variants of SARS-CoV-2, one must take the structures of the virus into consideration. One structure that is particularly important is the $\mathrm{S}$ structure responsible for the spike proteins that is responsible for the virus's ability to enter host cells and is considered the main target of most vaccines (Cevik et al., 2021). Despite the $S$ structure being the main target, it is not the only target as the vaccines are produced in such a way to incorporate various antibodies and T cells to reduce the risk of a single mutation reducing the efficacy and effectiveness of the vaccine drastically (Cevik et al., 2021).

It is worth noting that immunity towards the vaccine should theoretically take a long time. This is mainly due to the fact that the vaccine is acting to prevent infection rather than treating it (Cevik et al., 2021).

As for the interactions of the vaccine to the various variants, it has been seen that certain amino acid substitutions that occur due to mutations within the $\mathrm{S}$ structure receptor-binding domain and well $\mathrm{N}$-terminal domain could negatively affect the effectiveness of the vaccines when it comes to antibody neutralization (Cevik et al., 2021). 
For one, the deletion of the N-terminal domain (NTD) H69/V70, according to a study conducted by Rees-Spear et al., does not affect the neutralization potency of most mAbs (cross-neutralizing monoclonal antibodies) (Rees-Spear et al., 2021). However, the N501Y mutation which is shared across multiple variants removes almost all neutralizing activity of mAbs.

Another mutation that is similar to N501Y and could negatively affect the effectiveness and efficacy of vaccines is E484K, which occurs in Beta and Gamma variants. For this mutation, the reduction for the Beta variant was seen to be the biggest, with Gamma being only moderately affected. It is worth noting that Alpha variants do not have this E484K mutation, which could possibly explain why vaccine efficacy and effectiveness is higher for said variant; the Alpha variant shows a slight decrease when tested for antibody neutralization in in-vitro utilizing Oxford/AstraZeneca and Pfizer-BioNTech (Cevik et al., 2021). As for the Delta variant, similar to the Alpha variant, it does not contain the E484k mutation, and as such showcases a slight reduction in the antibody neutralization when tested against Oxford/AstraZeneca and Pfizer-BioNTech. However, it seems that inactivated vaccines (such as Sinovac and Sinopharm) are capable of neutralizing the E484K and N501Y mutations (Tregoning et al., 2021).

Overall, it seems that variants with the mutation N501Y, as well as E484K, result in a lower antibody neutralization, and that those with both see the most reduction in efficacy and effectiveness, as seen in Beta and Gamma when compared to the other variants (Cevik et al., 2021; Lucas et al., 2021).

\section{Conclusion}

In the Alpha variant, four mutations need to be considered because they affect the characteristics of the virus, causing an increase in cleavage of the S1/S2 and in turn increasing the infection rate. However, the Alpha variant does not drastically reduce the efficacy of the vaccines. Moreover, the Delta variant mutations can reduce immunity and increase replication, making it easier to spread which could explain why it is the most common variant in Indonesia. Despite this, the efficacy and effectiveness of the vaccine is only slightly reduced by Delta. As for the Beta variant, it was observed that the virus's mutations can bind to the neutralizing antibody, affecting the work of the antibodies and thus reducing the efficacy of the vaccines at a higher level than the other variants.

As for the local variants B.1.470 and B.1.466.2, they were seen to have several shared mutations with other the variants (Alpha, Beta, and Delta variants), with these mutations increasing the infection rate as well as enhancing the replication of the virus. However, one mutation that can be observed in the B.1466.2 variant may cause more infections, and that mutation is N439K. This mutation can bind to the hACE2 with higher affinity than the wild-type and can also neutralize antibodies of the vaccines.

And finally, the isolates utilized in this paper were acquired as early as the end October 2021 up until the end of November 2021. It should be noted that the classification of the isolates is not set in stone and is constantly changing with new findings as many isolates are constantly being reclassified on GISAID.org.

\section{Declarations}

\section{Acknowledgments}

The authors would like to express their gratitude to the Kholis Abdurachim Audah in supervising and designing this study.

\section{Authors' contributions}

DY, MJ, and NS have had equal contribution in this article. KAA have designed and supervised the study. DY, MJ and NS conducted WGS, analyzed the data, and written the manuscripts. All authors have read and approved the manuscript.

\section{Funding}


Our gratitude goes to Swiss German University and KAA for providing us with supports and funding.

\section{Availability of data and materials}

The datasets used and/or analyzed during the current study are available from the GISAID website.

\section{Ethics approval and consent to participate}

All study protocols were approved by National Institute of Health Research and Development MoH Indonesia ethics committee with the reference number of LB.02.01/5.2/KE 153. Signed informed consent was obtained from all patients. All methods were carried out in accordance with relevant guidelines and regulations.

\section{Consent for publication}

Not applicable.

\section{Competing interests}

We declare that there are no any competing interests.

\section{Author details}

${ }^{1}$ Department of Biomedical Engineering, Faculty of Life Sciences and Technology, Swiss German University, Tangerang 15143, Indonesia

${ }^{2}$ Academic Research and Community Service, Swiss German University, Tangerang 15143, Indonesia

\section{References}

Baum, D. (2008). Reading a phylogenetic tree: the meaning of monophyletic groups. Nature Education, 1(1), 190.

Cevik, M., Grubaugh, N. D., Iwasaki, A., \& Openshaw, P. (2021). COVID-19 vaccines: Keeping pace with SARS-CoV-2 variants. Cell, 184(20), 5077-5081.

Chrysostomou, A. C., Vrancken, B., Koumbaris, G., Themistokleous, G., Aristokleous, A., Masia, C., et al. (2021). A Comprehensive Molecular Epidemiological Analysis of SARS-CoV-2 Infection in Cyprus From April 2020 to January 2021: Evidence of a Highly Polyphyletic and Evolving Epidemic. Viruses 13, 1098. doi: 10.3390/v13061098

Clinic, M. (2021). Coronavirus disease 2019 (COVID-19). https://www.mayoclinic.org/diseasesconditions/coronavirus/symptoms-causes/syc-20479963.

Fibriani, A., Stephanie, R., Alfiantie, A. A., Siregar, A. L. F., Pradani, G. A. P., Yamahoki, N., .. \& Ristandi, R. B. (2021). Analysis of SARS-CoV-2 Genomes from West Java, Indonesia. Viruses, 13(10), 2097.

Gisaid - Initiative. (n.d.). Retrieved December 6, 2021, from https://www.epicov.org/epi3/frontend\#1c9f27.

La Rosa, G., Brandtner, D., Mancini, P., Veneri, C., Bonanno Ferraro, G., Bonadonna, L., ... \& Suffredini, E. (2021). Key SARSCoV-2 Mutations of Alpha, Gamma, and Eta Variants Detected in Urban Wastewaters in Italy by Long-Read Amplicon Sequencing Based on Nanopore Technology. Water, 13(18), 2503.

Liu, Y., Liu, J., Johnson, B. A., Xia, H., Ku, Z., Schindewolf, C., Widen, S. G., An, Z., Weaver, S. C., \& Menachery, V. D. (2021). Delta spike P681R mutation enhances SARS-CoV-2 fitness over Alpha variant. BioRxiv. 
Lubinski, B., Tang, T., Daniel, S., Jaimes, J. A., Whittaker, G. R. (2021). Functional Evaluation of Proteolytic Activation for the SARS-CoV-2 Variant B.1.1.7: Role of the P681H Mutation. bioRxiv. doi: 10.1101/2021.04.06.438731

Lucas, C., Vogels, C. B., Yildirim, I., Rothman, J. E., Lu, P., Monteiro, V., ... \& Iwasaki, A. (2021). Impact of circulating SARS-CoV-2 variants on mRNA vaccine-induced immunity. Nature, 1-7.

Tegally, H., Wilkinson, E., Giovanetti, M., Iranzadeh, A., Fonseca, V., Giandhari, J., ... \& de Oliveira, T. (2021). Detection of a SARS-CoV-2 variant of concern in South Africa. Nature, 592(7854), 438-443.

Masters, P. S. (2006). The molecular biology of coronaviruses. Advances in virus research, 66, 193-292.

Mittal, A., Manjunath, K., Ranjan, R. K., Kaushik, S., Kumar, S., \& Verma, V. (2020). COVID-19 pandemic: Insights into structure, function, and hACE2 receptor recognition by SARS-CoV-2. PLoS pathogens, 16(8), e1008762.

Mohammadi, M., Shayestehpour, M., \& Mirzaei, H. (2021). The impact of spike mutated variants of SARS-CoV2 [Alpha, Beta, Gamma, Delta, and Lambda] on the efficacy of subunit recombinant vaccines. Brazilian Journal of Infectious Diseases, 25.

Nieto-Torres, J. L., DeDiego, M. L., Álvarez, E., Jiménez-Guardeño, J. M., Regla-Nava, J. A., Llorente, M., ... \& Enjuanes, L. (2011). Subcellular location and topology of severe acute respiratory syndrome coronavirus envelope protein. Virology, $415(2), 69-82$.

Ostrov, D. A. (2021). Structural Consequences of Variation in SARS-CoV-2 B.1.1.7. J. Cell Immunol. 3, 103-108. doi: 10.33696/immunology.3.085

Rees-Spear, C., Muir, L., Griffith, S. A., Heaney, J., Aldon, Y., Snitselaar, J. L., ... \& SAFER Investigators. (2021). The effect of spike mutations on SARS-CoV-2 neutralization. Cell reports, 34(12), 108890.

Sanches, P. R., Charlie-Silva, I., Braz, H. L., Bittar, C., Calmon, M., Rahal, P., \& Cilli, E. M. (2021). Recent advances in SARS-CoV2 Spike protein and RBD mutations comparison between new variants Alpha (B. 1.1. 7, United Kingdom), Beta (B. 1.351, South Africa), Gamma (P. 1, Brazil) and Delta (B. 1.617. 2, India). Journal of Virus Eradication, 100054.

Socher, E., Conrad, M., Heger, L., Paulsen, F., Sticht, H., Zunke, F., et al. (2021). Mutations in the B.1.1.7 SARS-CoV-2 Spike Protein Reduce Receptor-Binding Affinity and Induce a Flexible Link to the Fusion Peptide. Biomedicines 9, 525. doi: 10.3390/biomedicines 9050525

Starr, T. N., Greaney, A. J., Hilton, S. K., Ellis, D., Crawford, K. H. D., Dingens, A. S., et al. (2020). Deep Mutational Scanning of SARS-CoV-2 Receptor Binding Domain Reveals Constraints on Folding and ACE2 Binding. Cell 182, 1295-1310.e1220. doi: 10.1016/j.cell.2020.08.012

Timmers, L.F.S.M., Peixoto, J.V., Ducati, R.G. et al. SARS-CoV-2 mutations in Brazil: from genomics to putative clinical conditions. Sci Rep 11, 11998 (2021). https://doi.org/10.1038/s41598-021-91585-6

Tracking SARS-CoV-2 Variants. (n.d.). WHO. https://www.who.int/en/activities/tracking-SARS-CoV-2-variants/

Tregoning, J. S., Flight, K. E., Higham, S. L., Wang, Z., \& Pierce, B. F. (2021). Progress of the COVID-19 vaccine effort: viruses, vaccines and variants versus efficacy, effectiveness and escape. Nature Reviews Immunology, 1-11.

WHO. (2020). Report of the WHO-China Joint Mission on Coronavirus Disease 2019 (COVID-19).

https://www.who.int/docs/default-source/coronaviruse/who-china-joint-mission-on-covid-19-finalreport.pdf\#: :text=People with COVID-19,mild disease and recover

Page $11 / 14$ 
Wiranti Wiranti, Ayun Sriatmi, W. K. (2020). Determinan Kepatuhan Masyarakat Kota Depok terhadap Kebijakan Pembatasan Sosial Berskala Besar dalam Pencegahan Covid-19. 9. https://jurnal.ugm.ac.id/jkki/article/view/58484

World Health Organization. (n.d.). Tracking sars-COV-2 variants. World Health Organization. Retrieved December 1, 2021, from https://www.who.int/en/activities/tracking-SARS-CoV-2-variants/

Wu, A., Peng, Y., Huang, B., Ding, X., Wang, X., Niu, P., ... \& Jiang, T. (2020). Genome composition and divergence of the novel coronavirus (2019-nCoV) originating in China. Cell host \& microbe, 27(3), 325-328.

Zhao, L. P., Lybrand, T. P., Gilbert, P. B., Hawn, T. R., Schiffer, J. T., Stamatatos, L., et al. (2021). Tracking SARS-CoV-2 Spike Protein Mutations in the United State/01 - 2021/03) Using a Statistical Learning Strategy. bioRxiv. doi:

$10.2139 /$ ssrn.3844900

Zhou, W., Xu, C., Wang, P., Luo, M., Xu, Z., Cheng, R., ... \& Jiang, Q. (2021). N439K variant in spike protein alter the infection efficiency and antigenicity of SARS-CoV-2 based on molecular dynamics simulation. Frontiers in cell and developmental biology, 2071.

\section{Table 3}

Table 3 is available in the Supplemental Files section

\section{Figures}

\section{Total Isolated Sequences of Alpha Variant in Indonesia}

\section{0}

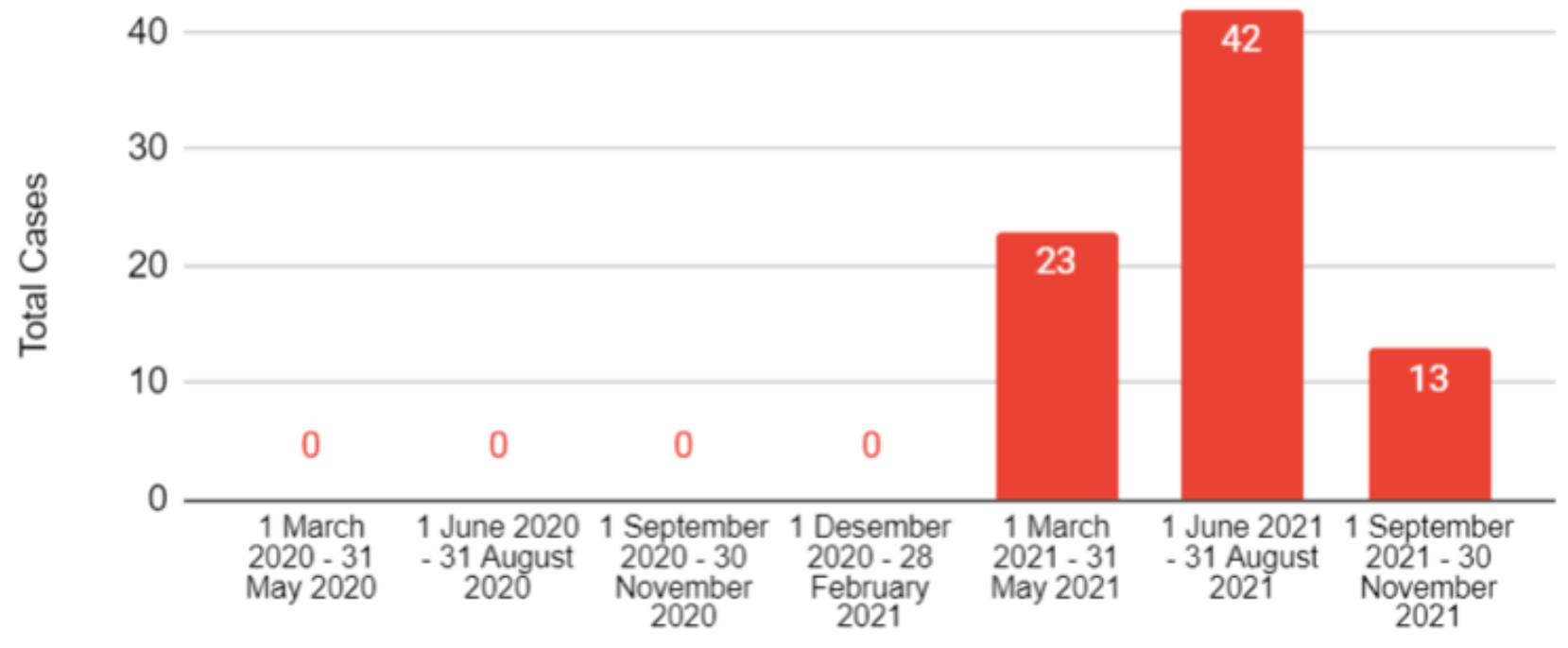

Time Period

\section{Figure 1}

Total Isolated Sequences of Alpha Variant in Indonesia 


\section{Total Isolated Sequences of Beta Variant in Indonesia}

15

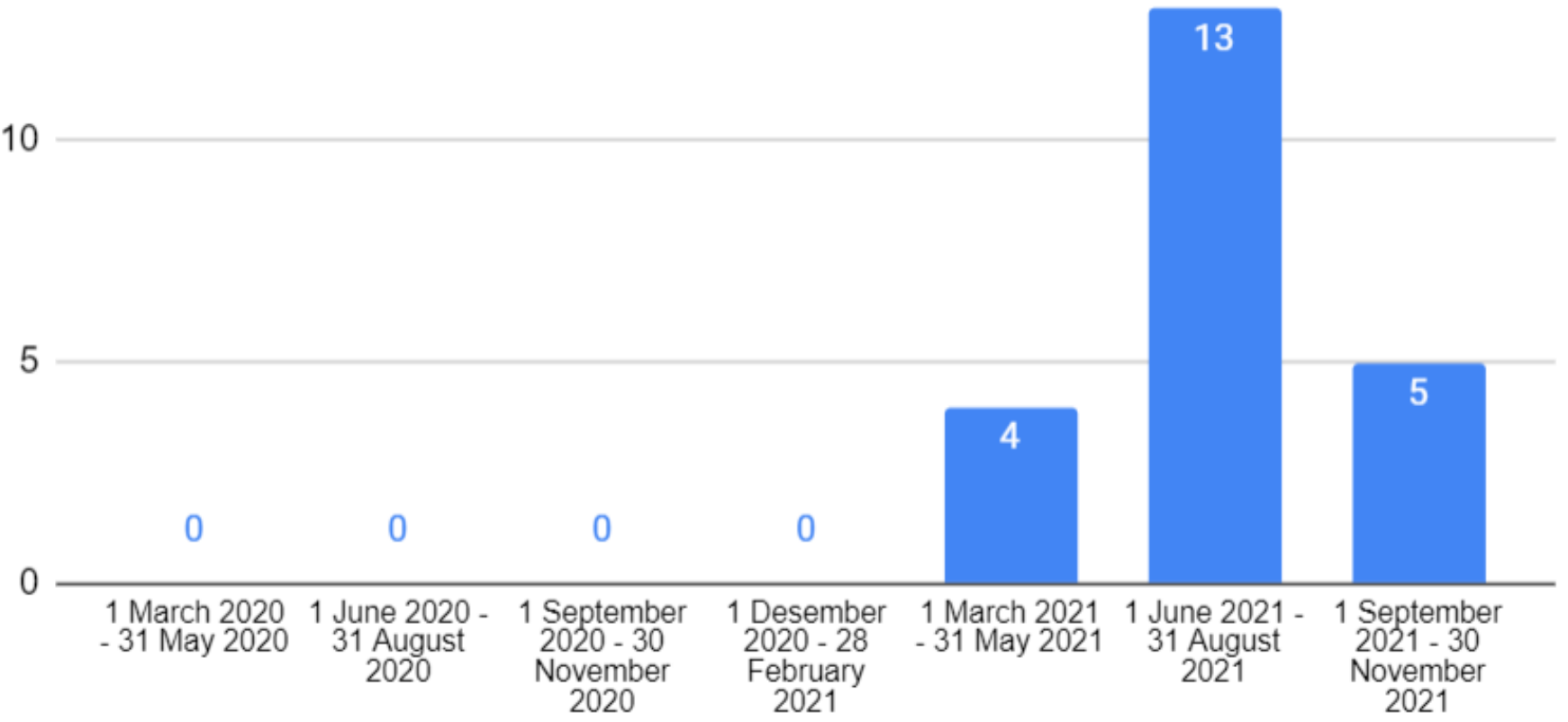

Time Period

Figure 2

Total Isolated Sequences of Beta Variant in Indonesia

Total Isolated Sequences of Delta Variant in Indonesia 4000

3000

\section{5}

2000

2230

焉

1000

\begin{tabular}{cccc}
0 & 0 & 0 & 0 \\
\hline 1 March 2020 & 1 June 2020 & 1 September & 1 Desember \\
-31 May & 31 August & $2020-30$ & $2020-28$ \\
2020 & 2020 & November & February \\
& & 2020 & 2021 \\
& & & Time Period
\end{tabular}




\section{Figure 3}

Total Isolated Sequences of Delta Variant in Indonesia

\section{Supplementary Files}

This is a list of supplementary files associated with this preprint. Click to download.

- Table3.docx

- SupplementaryFilesSARSCoV2variantsgenomeanalysisofIndonesianisolatesandtheirresponsestoavailablevaccines.pdf 